\title{
Photon emission from silver particles induced by a high-energy electron beam
}

\author{
N. Yamamoto and K. Araya \\ Department of Physics, Tokyo Institute of Technology, Meguro-ku, Tokyo 152-8551, Japan \\ F. J. García de Abajo \\ Centro Mixto CSIC-UPV/EHU and Donostia International Physics Center (DIPC), San Sebastián, Spain
}

(Received 11 July 2000; published 6 November 2001)

\begin{abstract}
Photon emission spectra from submicron silver particles induced by an electron beam have been measured using a light detection system combined with a $200-\mathrm{kV}$ transmission electron microscope. Multiple peaks appear in the spectra associated with collective plasmon excitations produced in the particles by the incident electrons. The wavelengths of these peaks are observed to shift towards larger values with increasing particle diameter, as predicted by Mie theory. Moreover, photon maps have been obtained in a scanning mode and they indicate that those peaks correspond to the multipole modes of electromagnetic oscillations in metallic spheres (Mie resonances). The spectral shape of the emission and the dependence on particle size, impact parameter, and electron energy are well explained from theoretical calculations of the photon emission probability derived from a fully retarded analytical treatment of the interaction of fast electrons with metallic spheres.
\end{abstract}

DOI: 10.1103/PhysRevB.64.205419

PACS number(s): 73.20.Mf, 41.20.Jb, 68.37.-d

\section{INTRODUCTION}

Photon emission induced by the passage of a fast electron through a material can occur via different mechanisms that include phenomena such as cathodoluminescence, Cherenkov radiation, and transition radiation. The emission can occur even when the electron passes near the material without actually going through it. This is the case of the SmithPurcell effect, ${ }^{1}$ where an electron beam passing parallel to the surface of a grating produces light emission of wavelength determined by the direction of emission and the spacing of the grating. ${ }^{2}$ Undulators in synchrotrons and freeelectron lasers rely on similar principles. A common feature to these forms of photon emission is that the incoming electrons produce induced currents on the objects with which they interact, and these currents cannot follow the motion of the electrons, and therefore, they can be regarded as accelerated induced charges that give rise to the emission of radiation.

A similar effect is investigated in the present work by combining a light detection system with a transmission electron microscope (TEM). The sample under study is formed by isolated submicron silver particles deposited on a thin carbon film. Light is emitted by interaction of the fast electrons of the microscope with the silver particles, and the collected emission spectra are discussed and compared with theoretical calculations below.

Our experimental setup combines the high spatial resolution of a TEM, which can focus an electron beam on a region of $1 \mathrm{~nm}$ in diameter at the position of the sample, and the high-energy resolution of a light detection system, below 1 $\mathrm{meV}$ in the optical region. This energy resolution is much better than that of other techniques commonly used in TEM, such as energy dispersive x-ray spectroscopy (EDX) and electron energy loss spectroscopy (EELS). This system has been developed as a useful tool to study optical properties of semiconductors and dielectrics. In particular, the TEMcathodoluminescence technique makes it possible to evaluate optical behavior of crystal defects and nanostructures in semiconductors.

The authors have intensively studied photon emission and transition radiation from thin films and metallic microstructures using this technique, ${ }^{3-6}$ and have found that the emission spectra are satisfactorily explained from the theory when the sample has a regular shape with a flat surface. ${ }^{3-5}$ However, the edges and surface roughness resulted in additional structures in the emission spectra, which were clearly revealed in monochromatic photon maps obtained in a scanning mode, showing high sensitivity of the emission to the shape of the sample.

Recently, we have observed some anomalous features in the light emission from gold and silver particles of $\sim 100 \mathrm{~nm}$ in diameter. ${ }^{6}$ Their spectra are characterized by multiple peaks whose relative intensities change with the position of the electron beam, which is focused on the target. In a first attempt to explain the wavelengths of those peaks, one can consider the resonance frequencies of plasma oscillations in small metallic spheres, which are subject to the relation $\varepsilon\left(\omega_{1}\right)=-(l+1) / l$, where $\varepsilon(\omega)$ is the macroscopic dielectric function of the metal and $l$ is the order of the mode. Using the dielectric function of silver, the wavelengths obtained from this equation corresponding to the resonance frequencies are distributed in a narrow range that goes from $338 \mathrm{~nm}(l=\infty)$ to $354 \mathrm{~nm}(l=1)$. However, the observed peaks appear at longer wavelengths, which cannot be explained by the above equation for the plasma oscillation frequencies in a sphere. Actually, retardation effects show up when the wavelength of a given mode is comparable in magnitude to the sphere diameter, so that a retarded description of the sphere response is needed, as the one presented below. The above equation is no longer appropriate, and one has to use a retarded version to account for the so-called Mie resonances. ${ }^{7}$

In a related context, the energy loss probability of an electron passing near an isolated sphere has been studied by EELS using scanning transmission electron microscopes 
(STEM). Photon emission contributes to the electron energy loss process, and thus, the emission spectrum is closely related to EELS. A number of experimental studies by STEM have been performed for metal and dielectric single particles, ${ }^{8-11}$ and nonretarded theoretical analyses explained relatively well those EELS spectra for small particle sizes. ${ }^{12-15}$ However, retardation corrections become increasingly important as higher electron velocities and larger particle sizes are considered. Treatments of EELS from a sphere that include retardation effects have been given by Fujimoto and Komaki ${ }^{16}$ and more recently by García de Abajo and Howie ${ }^{17,18}$ who derived a close analytical expression for the radiation emission from a sphere in interaction with a fast electron.

In the present paper, observations of spectra of photon emission from silver particles induced by an electron beam are reported. The dependence of the observed emission peak wavelengths on beam position and particle size is discussed in the light of a fully retarded theoretical description of photon emission induced by fast electrons passing near a sphere. Such a theory is summarized in Sec. II, and the experimental setup is described in Sec. III. The emission peaks in the spectra can be associated with multipole mode oscillations of the electromagnetic field induced by the passage of the electrons, as is shown by analyzing the observed monochromatic photon maps offered in Sec. IV A. The emergence of multiple peaks and their dependence on particle size and accelerating voltage are discussed and compared with the theory in Secs. IV B and IV C. Finally, the main conclusions are summarized in Sec. V.

\section{THEORY}

Under the experimental conditions investigated in the present work, the emission of radiation due to the interaction between a metallic particle and an external electron originates in the coupling of the electron to collective plasmon modes of the particle. The observed particles have nearly spherical shapes, so that they will be described by perfect spheres. Besides, the particles are supported by a carbon film, thin enough as to be neglected in the calculations that follow.

In a nonretarded treatment, the energy of the sphere plasmons can be obtained by solving Poisson's equation, leading to the relation

$$
\varepsilon\left(\omega_{l}\right)=-(l+1) / l,
$$

where $\omega_{1}$ is the frequency of the plasmon modes associated with the orbital momentum number $l$ and $\varepsilon(\omega)$ is the frequency-dependent dielectric function of the material of which the sphere is made up. Equation (1) determines the position of the loss peaks in the electron energy loss spectra calculated within a nonretarded treatment of the sphereelectron interaction, as first given by Ferrell and Echenique. $^{12}$

Equation (1) is independent of the sphere size, and therefore, the resonance frequencies that it predicts are independent of that parameter as well. However, this is clearly inconsistent with the observed emission from silver particles

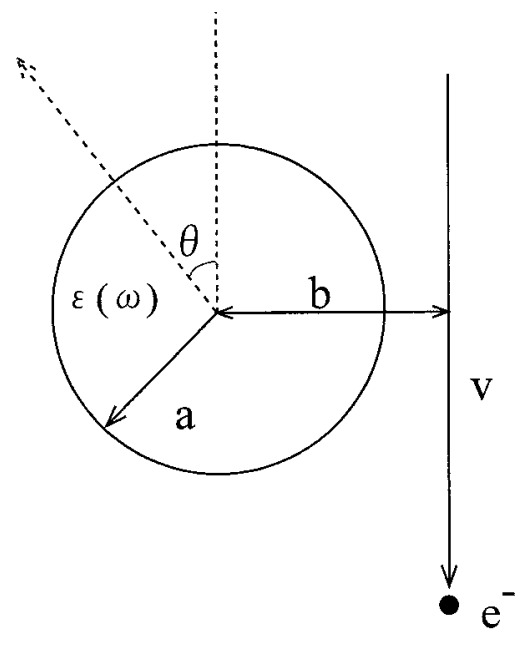

FIG. 1. Geometry of an incident electron passing near a metal sphere.

reported here, where the sphere diameters under consideration are of the order of $D=90-285 \mathrm{~nm}$ and the wavelengths of the emitted light are in the $\lambda=300-600 \mathrm{~nm}$ range. As a consequence, retardation effects can play a substantial role in the interaction between induced charges situated at opposite sides of the sphere, since the time needed to propagate the electromagnetic signal that communicates them $(\approx D / c)$ is of the same order of magnitude as the characteristic plasma oscillation periods $(\approx \lambda / c)$. Then, a retarded treatment of the interaction between the electron and the sphere capable of describing the well-known size-dependent Mie oscillations ${ }^{7}$ becomes necessary.

A fully retarded description of both the electron energy loss probability and the fast-electron-induced photon emission probability for the sphere has been recently developed by the authors. ${ }^{17,18}$ In it, the external electric field due to the passing electron is decomposed into multipole components with respect to the center of the sphere. Each multipole component is then scattered separately by the sphere, giving rise to a scattered or induced electric field, which acts back on the electron producing a retarding force responsible for its energy loss. The external electric field is evanescent and does not produce any radiation. However, the induced field gives rise to radiation in the far field, which is just the photon emission discussed here.

When the electron trajectory does not pass through the sphere, as shown in Fig. 1, the probability of photon emission takes the form (in atomic units)

$$
\Gamma^{r a d}(\omega)=\frac{1}{c \omega} \sum_{l=1}^{\infty} \sum_{m=-l}^{l} K_{m}^{2}\left(\frac{\omega b}{v \gamma}\right)\left[C_{l m}^{M}\left|t_{l}^{M}\right|^{2}+C_{l m}^{E}\left|t_{l}^{E}\right|^{2}\right],
$$

where $\omega$ is the photon frequency, $K_{m}$ is a modified Bessel function, $v$ is the electron velocity, $\gamma=1 / \sqrt{1-v^{2} / c^{2}}$ is the Lorentz factor accounting for the retarded reduction of the sphere oscillation frequency as seen from the frame of the incoming electron, and $b$ is the electron impact parameter with respect to the sphere center. The coefficients $C_{l m}^{M}$ and $C_{l m}^{E}$ inside the square brackets depend exclusively on the $v / c$ 
ratio, whereas $t_{l}^{M}$ and $t_{l}^{E}$ are scattering $t$ matrices for magnetic and electric modes in the sphere (see Ref. 18 for explicit expressions). The electric modes are dominant for metallic spheres and for the electron velocities under consideration $(v / c=0.7)$. Therefore, a given peak in an emission spectrum can be associated with a maximum of the scattering matrix element $t_{l}^{E}$ for a particular $l$ th-order mode. $t_{l}^{E}$ depends both on the sphere diameter $D$ and on the dielectric function $\varepsilon(\omega)$, but not on the electron velocity. Its poles must satisfy the expression ${ }^{7,18}$

$$
h_{l}^{(+)}\left(\rho_{0}\right) \frac{d\left[\rho_{1} j_{l}\left(\rho_{1}\right)\right]}{d \rho_{1}}=\varepsilon \frac{d\left[\rho_{0} h_{l}^{(+)}\left(\rho_{0}\right)\right]}{d \rho_{0}} j_{l}\left(\rho_{1}\right),
$$

where $h_{l}^{(+)}$is a spherical Hankel function, $\rho_{0}=\omega D / 2 c$, and $\rho_{1}=\rho_{0} \sqrt{\varepsilon}$ with $\operatorname{Im}\left\{\rho_{1}\right\}>0$. Equation (3) is the retarded version of Eq. (1), and it predicts a redshift of the plasmon frequencies with increasing diameter for metallic spheres. ${ }^{18}$ In addition, the frequencies derived from Eq. (3) have in general a negative imaginary part that gives rise to a finite natural width in the emission features. For small particles (i.e., $\rho_{0} \ll 1$ ), Eq. (3) reduces to

$\varepsilon^{2} \frac{l x}{2 l+3}+\varepsilon\left[\frac{3(2 l+1) x}{(2 l+3)(2 l-1)}-1\right]-(l+1)\left(1+\frac{x}{2 l-1}\right)=0$,

where $x=\rho_{0}^{2} / 2$.

For a penetrating trajectory, Eq. (2) has to be supplemented by the contribution to the emission coming from the part of the trajectory contained within the sphere. The corresponding expression is more involved, but the peak structure is still dominated by the poles given by Eq. (3).

\section{EXPERIMENT}

Silver particles were produced by evaporation in an $\mathrm{Ar}$ atmosphere and were collected on a thin amorphous carbon film supported by a copper mesh of $3 \mathrm{~mm}$ in diameter. Photon emission spectra were acquired by a light detection system combined with a transmission electron microscope operated at an accelerating voltage of $200 \mathrm{kV}$. The light emitted from individual Ag particles is collected by an ellipsoidal mirror above a specimen holder and is focused on the entrance slit of a monochrometer outside the TEM.

We used a standard TEM (JEM-2000FX) in this work, which is slightly modified around the pole piece for the insertion of the ellipsoidal mirror. The TEM is of analytical type and has a pole piece with a wide gap $(15 \mathrm{~mm})$. The source is a $\mathrm{LaB}_{6}$ filament.

The acceptance angles of the mirror lie in the range from $\theta=3.6^{\circ}-84^{\circ}$ (see Fig. 1). A linear polarizer is located between the ellipsoidal mirror and the monochrometer to select linearly polarized components of the emitted light. Then $p$ and $s$ polarization can be measured independently. The emission spectra shown below are not corrected for frequencydependent detection efficiency, and the intensity decreases considerably in the wavelength range below $300 \mathrm{~nm}$ and above $800 \mathrm{~nm}$ due to the detection efficiency of the system.
The size of the electron beam at the position of the target is $10 \mathrm{~nm}$ in diameter. This is a relatively large value compared with the sizes of $\sim 1 \mathrm{~nm}$ usually employed in scanning electron microscopy (SEM) imaging in the TEM. The reason for this was to obtain higher beam currents and, therefore, a sufficient photon count. However, the beam diameter is still much smaller than the size of the particles (90-285 nm). Nevertheless, a smaller probe size was used to obtain images in the SEM mode (see Sec. IV A below). The measured probe current is $\sim 0.2 \mathrm{nA}$ at $200 \mathrm{kV}$ using the same lens and current conditions as in the SEM mode. The particle sizes were measured from SEM images, with a scale calibrated using a standard sample.

Transition radiation coming from the carbon film, radiation coming from the gun filament, and stray light can contribute to the background in the spectra. However, we have found that those background light intensities are very small in comparison with the signal intensity coming from Ag particles when a high beam current is used in our setup.

Monochromatic photon maps have been obtained by scanning the electron probe over the sample region in the SEM mode. Then, an image is composed of, for example, 100 $\times 100$ pixels, so that the focused electron beam is scanned across the specimen and stays $0.1 \mathrm{sec}$ for each pixel. Therefore, it takes $1000 \mathrm{sec}$ to obtain one complete photon map. The intensity in each pixel corresponds to an integrated intensity of the detected light during the dwell time of the beam ( $0.1 \mathrm{sec}$ in this case). So this imaging technique is similar to SEM or EDX mapping, except that we use light intensity collected by an ellipsoidal mirror, instead of secondary-electron yields as in the case of SEM. The acceptance solid angle of the mirror is the same both for photon map imaging and for the acquisition of spectra.

\section{RESULTS AND DISCUSSION}

\section{A. Emission spectra and photon maps}

Figure 2 shows emission spectra of $p$-polarized light from an $\mathrm{Ag}$ particle of $140 \mathrm{~nm}$ in diameter taken with the electron probe located at various positions $(A-D)$ as is schematically illustrated in Fig. 2. Two prominent peaks appear in each spectrum: a sharp peak at $360 \mathrm{~nm}$ and a broad peak at 420 $\mathrm{nm}$. The intensities of these peaks are enhanced when the probe position is near the sphere edge (positions $B$ and $C$ ). The wavelengths of these peaks show little variation when moving the electron probe position. The wavelength of the sharp peak is seen to slightly change from 357 to $363 \mathrm{~nm}$ by shifting the probe position from the center to the outside of the particle, i.e., when moving from $A$ to $D$.

Figure 3(a) is a SEM image of the same Ag particle as above. Figures 3(b)-3(d) are monochromatic photon maps taken at the peak wavelengths using different polarization directions: (b) and (c) are $p$ - and $s$-polarized photon maps, respectively, taken at a wavelength of $420 \mathrm{~nm}$, and (d) and (e) are $p$ - and $s$-polarized photon maps, respectively, taken at a wavelength of $360 \mathrm{~nm}$.

In Fig. 3(b), a pair of bright regions shows up along the edge of the particle in the polarization direction indicated by $\mathbf{E}$, which is parallel to the longer axis of the ellipsoidal mir- 


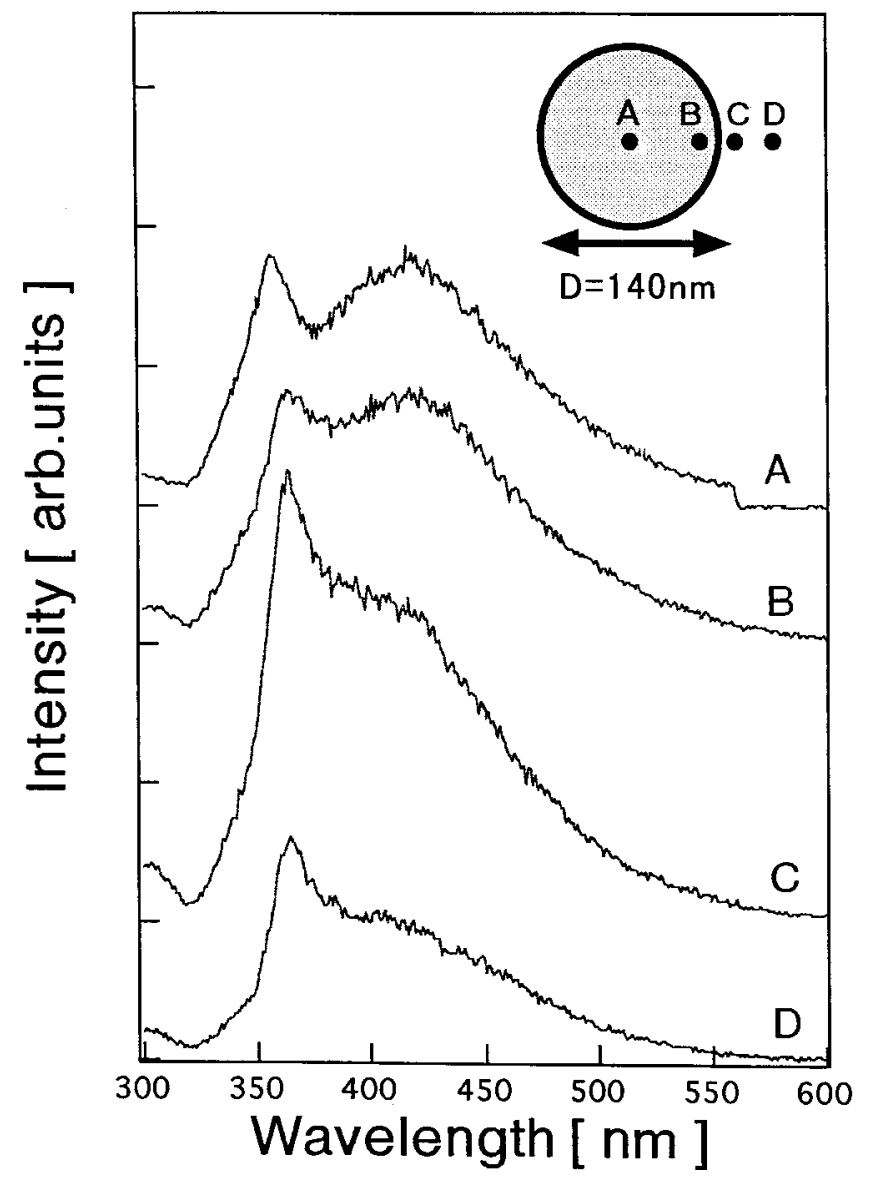

FIG. 2. Emission spectra from a silver particle of $140 \mathrm{~nm}$ in diameter taken at various positions of the electron beam probe. The emission collection angles range from $\theta=3.6^{\circ}$ to $84^{\circ}$ all throughout this paper (see Fig. 1). The electron accelerating voltage is $200 \mathrm{kV}$.

ror. When rotating the polarization direction by $90^{\circ}$, the orientation of the bright regions rotates $90^{\circ}$ as well, as seen in Fig. 3(c). This clearly suggests that the emission is caused by the electromagnetic oscillation of the dipole mode: the electric dipole induced in the particle by the passing electron is oriented towards the electron trajectory when the electron passes near the particle edge, and thus, the electric field of the light which is emitted due to the oscillation of this electric dipole is mainly polarized along the direction parallel to the plane determined by the particle center and the electron trajectory.

In Fig. 3(d), four bright regions are seen to appear along the edge of the particle in the directions parallel and perpendicular to the polarization direction of the detected light indicated by $\mathbf{E}$. Therefore, this emission is caused by the electromagnetic oscillation of the quadrupole mode. The orientation of the four bright regions is unchanged by rotation of the polarization direction by $90^{\circ}$, as one can see by comparing Figs. 3(d) and 3(e).

Figure 4 shows spectra of the $p$-polarized component of light emitted from an $\mathrm{Ag}$ particle of $180 \mathrm{~nm}$ in diameter taken at various probe positions as in Fig. 2. A single broad peak appears around $390 \mathrm{~nm}$. The peak intensity has a maximum when the probe position is located near the particle
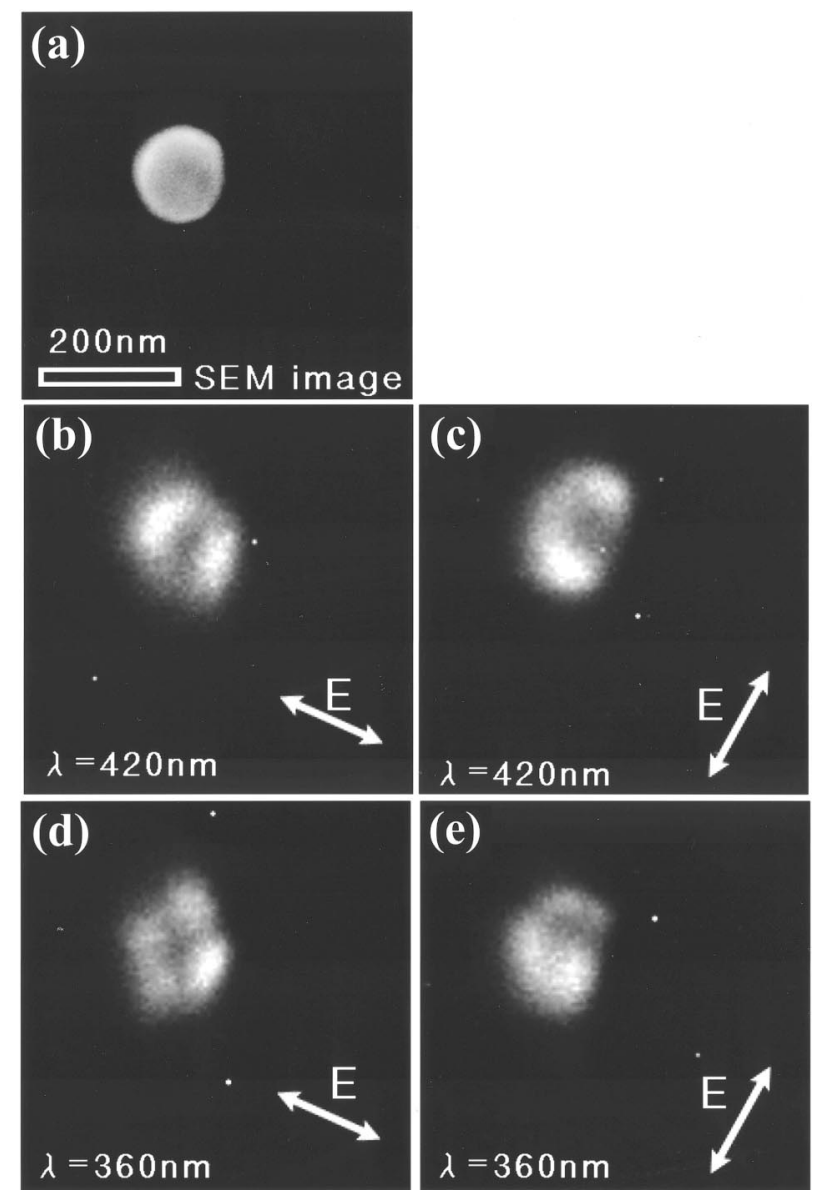

FIG. 3. (a) Secondary-electron emission (SEM) image of a silver particle of $140 \mathrm{~nm}$ in diameter. (b)-(e) Photon images of the same particle taken with linearly polarized light induced by fast electrons and for two polarization directions, as indicated by the polarization vector $\mathbf{E}$ in the images. Each pixel in the image corresponds to a different position of the electron beam and the intensity of the pixel is made proportional to the emission intensity for light polarized along the directions shown by the arrows. The photon images of (b) and (c) were taken at a wavelength of $420 \mathrm{~nm}$, whereas those of (d) and (e) were taken at $360 \mathrm{~nm}$. Dark (bright) regions correspond to low (high) photon emission intensity. The electron accelerating voltage is $200 \mathrm{kV}$.

edge $(B$ and $C)$. The peak wavelength shifts slightly from 378 to $395 \mathrm{~nm}$ when moving the probe position from $A$ to $D$.

In the measurement of the spectra, attention must be paid to several factors that can affect the spectral shape. In particular, deformation of the particle shape from an ideal sphere and also adsorption of fine particles on the particle can change the peak wavelength and spectral shape.

\section{B. Size dependence}

The emission spectrum is very sensitive to the size of the silver particle as one can see in Figs. 2 and 4. Figure 5(a) shows emission spectra of the $p$-polarized light component in the emission coming from silver particles with various diameters ranging from 90 to $285 \mathrm{~nm}$. The electron probe was located slightly outside the edge of the particles in all cases. 


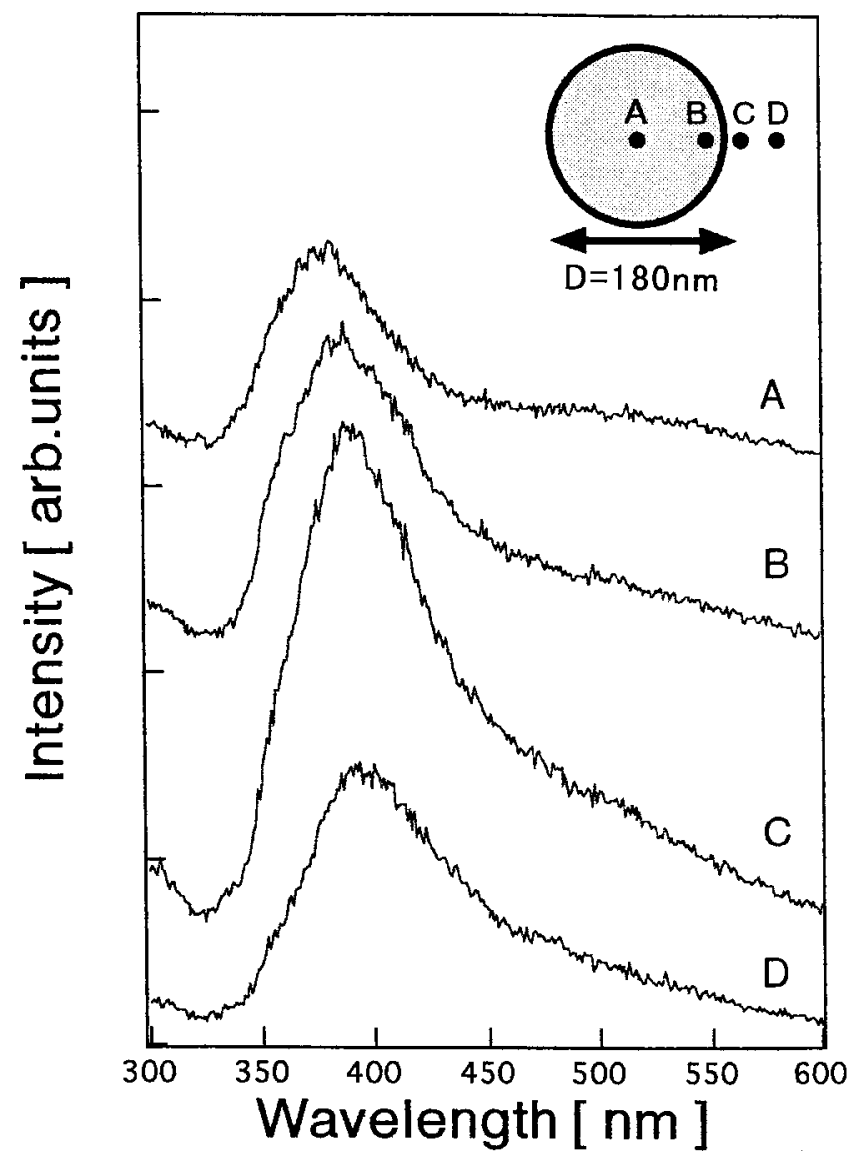

FIG. 4. Emission spectra from a silver particle of $180 \mathrm{~nm}$ in diameter taken at various probe positions. The electron accelerating voltage is $200 \mathrm{kV}$.

One can clearly see that the emission peaks shift to longer wavelengths with increasing particle diameter. There are at least three series of emission peaks as indicated by different marks in Fig. 5. A particle with a diameter of $90 \mathrm{~nm}$ exhibits just a single peak belonging to series 1 (dipole peak, marked by solid circles). When increasing the particle size, a peak of series 2 (quadrupole peak, marked by solid squares) appears in the spectra, and then two peaks of series 1 and 2 exist for particles with diameters of 140 and $155 \mathrm{~nm}$. By further increasing the particle size, only a single peak of series 2 is dominant for a diameter of $180 \mathrm{~nm}$, and then a peak of series 3 (hexapole peak, marked by solid triangles) shows up for particles diameters of $210-285 \mathrm{~nm}$, together with the peak of series 2.

The emission spectra shown in Fig. 5(b) were calculated using the theory described in Sec. II for the same particle diameters as in Fig. 5(a). The light emission is collected in the backward direction with respect to the propagation direction of the electron. The angle subtended by the ellipsoidal mirror is in the range from $\Theta=3.6^{\circ}$ to $84^{\circ}$, measured from the electron trajectory (see Fig. 1). The calculated emission intensity has been integrated over this angular range in order to compare with experiment. In the calculation, the electron moves $5 \mathrm{~nm}$ away from the sphere surface (i.e., $b=a$ $+5 \mathrm{~nm}$ ) and the dielectric function of silver has been taken from Ref. 19. There is a good correspondence in spectral

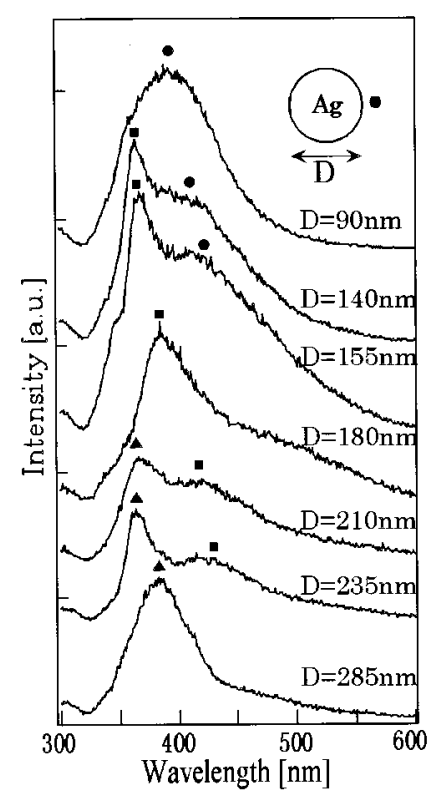

(a)

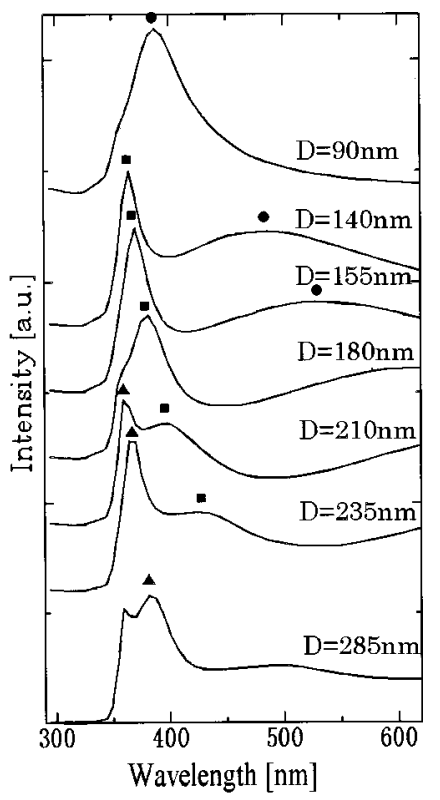

(b)
FIG. 5. (a) Emission spectra from silver particles of various diameters taken at an electron probe position slightly outside the particle edge. (b) Calculated spectra corresponding to those shown in (a). Three series of peaks appear in the spectra, which are marked by solid circles, squares, and triangles, corresponding to the dipole, quadrupole, and hexapole modes, respectively. The electron accelerating voltage is $200 \mathrm{kV}$.

shape and peak position between the observed and calculated spectra, as seen from Figs. 5(a) and 5(b). The calculated spectra reproduce reasonably well the fact that a single broad peak appears in the spectrum for a particle diameter of $90 \mathrm{~nm}$ and a sharp peak appears within a shorter-wavelength region for a particle diameter of $140 \mathrm{~nm}$. Moreover, those peaks shift to longer wavelengths with increasing particle diameter in the calculation. However, some discrepancy between Figs. 5(a) and 5(b) is apparent: the emission intensity of the dipole mode still has a finite intensity for the larger spheres in Fig. 5(b), though those peaks become weak for spheres with diameter larger than $155 \mathrm{~nm}$ in the observed spectra; besides, the peak wavelength of the dipole mode shifts towards longer wavelengths in the calculation as compared to the experiment. The discrepancy between experimental and theoretical spectra is partly due to a poor efficiency of the detection system in the long-wavelength region.

Each series of peaks is considered to belong to a different multipole mode of electromagnetic oscillation: that is, series 1 corresponds to the dipole mode $(l=1)$, series 2 to the quadrupole mode $(l=2)$, and series 3 to the hexapole mode $(l=3)$. In order to confirm this, we observed photon maps at these peak wavelengths. Figure 6 shows such photon maps of particles with different diameters: (a) and (b) are the same images as Figs. 3(d) and 3(b) for the particle with a diameter of $140 \mathrm{~nm}$, (c) is a photon map of the particle with a diameter of $180 \mathrm{~nm}$, and (d) and (e) are those obtained for a diameter of $230 \mathrm{~nm}$. Image (b), which was taken using the peak wavelength of series 1 , reveals a distribution characteristic of a dipolar oscillation mode, as mentioned above. Im- 


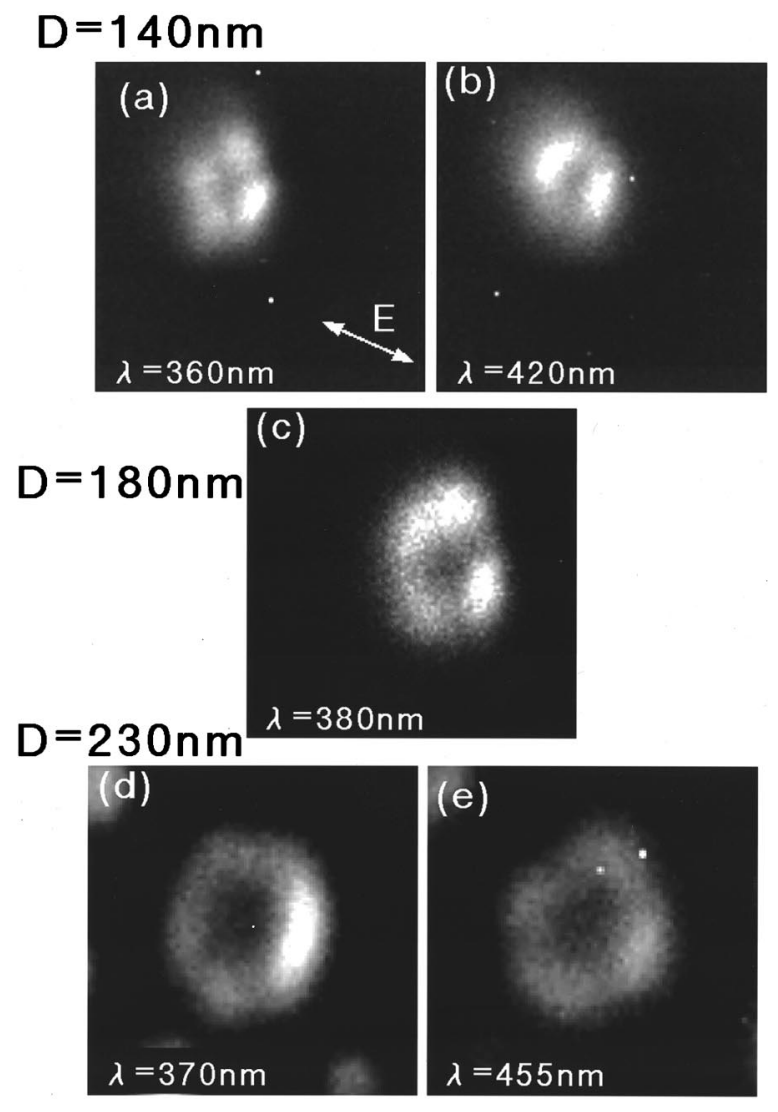

FIG. 6. Photon images of silver particles of three different diameters, namely, 140, 180, and $230 \mathrm{~nm}$, taken with $p$-polarized light. The light of wavelengths corresponding to the various peaks of the spectra shown in Fig. 5 was used to image the particles (see labels on the figures). The electron accelerating voltage is $200 \mathrm{kV}$.

ages (a), (c), and (e) were taken with the peak wavelengths of series 2 and have similar intensity distributions, showing four bright regions around the particle, which reveal the excitation of a quadrupolar mode. Finally, image (d), taken by using the peak wavelength of series 3 , shows six bright regions along the particle edge, which can be attributed to the excitation of hexapolar modes (some of the bright regions in this case are connected and this might complicate the interpretation of this image, but the positional arrangement of the bright regions definitely reveals the hexagonal symmetry, easily distinguished from the patterns of dipole and quadrupole modes). Therefore, these results strongly support the idea that the different series of peaks correspond to different multipole modes.

The energies corresponding to the peak wavelengths are plotted in Fig. 7 as a function of particle diameter (upper part). The peaks are classified into three groups corresponding to the multipole mode order $(l=1,2$, and 3$)$, which is indicated by the same marks as those used in Fig. 5. Each peak is clearly visible only within a finite range of diameters; the peak of the dipole mode appears for diameters below 180 $\mathrm{nm}$, that of the quadrupole mode is present in the range from 100 to $240 \mathrm{~nm}$, and that of the hexapole mode can be seen for diameters larger than $200 \mathrm{~nm}$. The peak energy decreases with increasing diameter, and this is very consistent with the

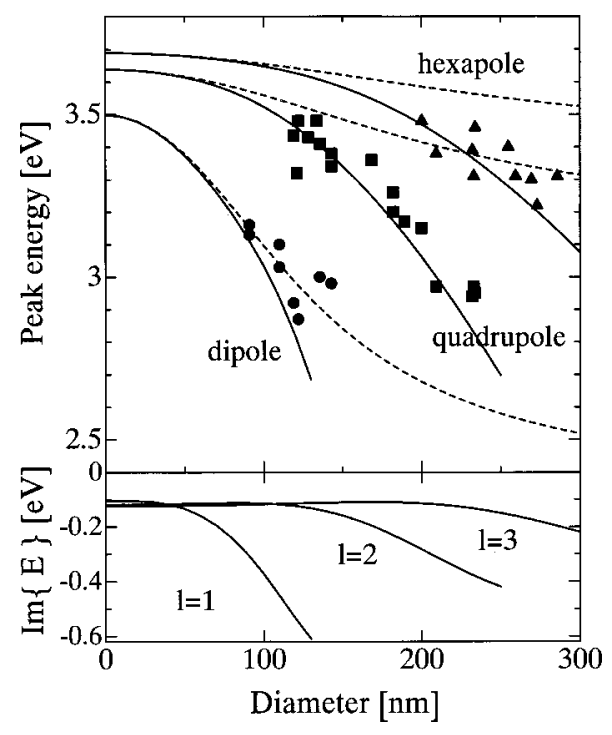

FIG. 7. Upper part: peak energies obtained from the emission spectra of silver particles as a function of particle diameter; the symbols correspond to the experimental results obtained from the spectra of Fig. 5(a) for $l=1$ (circles), $l=2$ (squares), and $l=3$ (triangles); the solid curves are theoretical results derived from the solution of Eq. (3) [this agrees well with the peak wavelengths of the spectra of Fig. 5(b)]; and the dashed curves stand for the approximate solution in the small-particle limit [Eq. (4)]. Lower part: imaginary part of the mode energies obtained from Eq. (3).

solid curves, corresponding to the theory, that have been calculated from Eq. (3). The peak energy values in the $D=0$ limit reduce to the one calculated from the nonretarded expression of Eq. (1) (more precisely, $\hbar \omega_{1}=3.503 \mathrm{eV}, \hbar \omega_{2}$ $=3.599 \mathrm{eV}$, and $\hbar \omega_{3}=3.625 \mathrm{eV}$ for silver). For finite values of $D$, the calculated mode energies of Fig. 7 (upper part) agree well with those of the peaks in the calculated spectra shown in Fig. 5. In particular, the resonant frequency of the dipole mode is strongly affected by retardation effects within the particle size under study, as compared to higher-order modes. A noticeable deviation from the nonretarded limit begins at $50 \mathrm{~nm}$ for the dipole mode, $100 \mathrm{~nm}$ for the quadrupole mode, and $150 \mathrm{~nm}$ for the hexapole mode. This can be ascribed to the different way in which retardation effects influence the different multipole modes. Dipole modes involve oscillations of the induced charge density on opposite sides of the sphere, so that their mutual electromagnetic interaction involves a distance of the order of the sphere diameter. The change in the phase of an electromagnetic signal of wavelength $\lambda=400 \mathrm{~nm}$ traveling in vacuum across a distance of $100 \mathrm{~nm}$ is $90^{\circ}$, so that for spheres of that size, whose dipole mode lies in that wavelength region, retardation is expected to play a dominant role for dipole modes. However, higher-order modes involve oscillations of the induced charge density with more nodes, so that the interaction between opposite sides of the sphere is reduced as compared to the dipole mode due to cancellation of contributions originating in positive and negative induced charges. Therefore, the relevant interaction involves smaller distances that make retardation effects weaker, and this is more so as the order of the mode increases. Following this argument, the diameter 
from which a mode $l$ deviates noticeably from the nonretarded result must scale approximately linearly with $l$, as it is actually the case.

The dashed curves in Fig. 7 are obtained from Eq. (4), which is an approximation to Eq. (3) for small particle diameters to second order in $\omega D / c$. This approximation gives reasonable values for particle diameters below $100 \mathrm{~nm}$. Most of the experimental data shown in the figure lie above that region and exhibit higher-order retardation corrections, which are fully accounted for by Eq. (3).

The lower part of Fig. 7 shows the imaginary part of the mode energies calculated from Eq. (4). This can be interpreted as an inverse lifetime that shows up in the spectra as a broadening of the peaks. Modes with $l=2$ and $l=3$ have a broadening of $\sim 0.12 \mathrm{eV}$, rather independent of particle diameter, and this corresponds to a lifetime of $\sim 2.7 \mathrm{fs}$. They appear to be sharper in the spectra of Fig. 5 than the dipole mode. The latter shows a steep increase in broadening with increasing particle diameter, and its lifetime becomes $\sim 0.8$ fs for $D=100 \mathrm{~nm}$. The broadening in wavelength can be estimated as $\Delta \lambda \approx \lambda \Delta \omega / \omega \approx 100 \mathrm{~nm}$ for $l=1$ and $D=100 \mathrm{~nm}$. This is in very good correspondence with the observed spectra of Fig. 5.

\section{Accelerating voltage dependence}

In this section we examine the effect of the accelerating voltage or electron velocity on the emission spectra and photon maps. Figure 8(a) shows emission spectra from an $\mathrm{Ag}$ particle with a diameter of $122 \mathrm{~nm}$ taken at accelerating voltages of (a) $100 \mathrm{kV}$ and (b) $200 \mathrm{kV}$, respectively. The probe position is slightly outside the edge as in Fig. 5, and $p$-polarized light is collected. Two peaks are seen to appear at wavelengths of 356 and $432 \mathrm{~nm}$ in those spectra. The broad peak at $432 \mathrm{~nm}$ corresponds to the dipole mode, and the sharp peak at $356 \mathrm{~nm}$ corresponds to the quadrupole mode. One can clearly see that the positions of the two peaks do not shift when changing the accelerating voltage. However, the relative intensities of the two peaks are seen to change in the sense that the dipole mode becomes stronger for higher accelerating voltages as compared to the quadrupole mode. Figure 8(b) shows calculated spectra corresponding to those in Fig. 8(a). The calculated spectra reproduce reasonably well the observed ones, showing that the increase in accelerating voltage produces no shift in the peak positions and at the same time it originates changes in the relative intensity of the two features that go in the same direction as in the experiment. The fact that the peak wavelength does not depend on accelerating voltage is consistent with the theoretical prediction of Eq. (2), since the information on the peak positions is fully contained in the scattering matrices, which are independent of the electron velocity. On the other hand, the relative intensities of the multipole modes depend on the accelerating voltage through the coefficients $C_{l m}^{E, M}$, which are a function of the velocity of the incident electron. The electronic modes dominate over the magnetic modes in the range from $100 \mathrm{kV}(v / c=0.55)$ to $200 \mathrm{kV}(v / c=0.70)$, and the coefficients $C_{l m}^{E}$ are roughly proportional to $\left[\left(c^{2}\right.\right.$ $\left.\left.-v^{2}\right) / v^{2}\right]^{l+1}$ within this velocity range (see Fig. 3 of Ref.
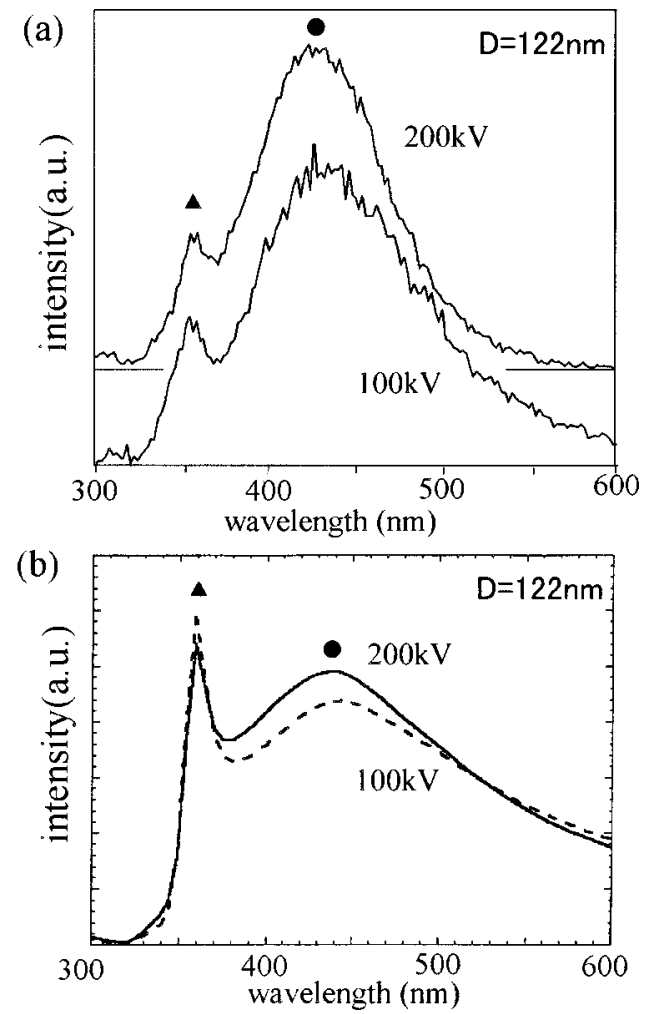

FIG. 8. (a) Emission spectra from a silver particle with a diameter of $122 \mathrm{~nm}$ taken for an electron accelerating voltage of 100 and $200 \mathrm{kV}$. (b) The corresponding calculated spectra for $100 \mathrm{kV}$ (dashed curve) and $200 \mathrm{kV}$ (solid curve).

18). Therefore the ratio of the radiative loss probability between the dipole mode and quadrupole mode must scales with the electron velocity as $\Gamma^{\text {dipole }} / \Gamma^{\text {quad }} \propto v^{2} /\left(c^{2}-v^{2}\right)$, and this ratio increases with velocity (i.e., with the accelerating voltage). This is consistent with the behavior observed in Fig. 8(a).

Figures 9(a) and 9(b) show photon maps of the same $\mathrm{Ag}$ particle as in Fig. 8, taken at (a) $100 \mathrm{kV}$ and (b) $200 \mathrm{kV}$, respectively, with a wavelength of $432 \mathrm{~nm}$. Each photon map shows two bright regions split along the polarization direction, indicating that they originate in light emission assisted by a dipole mode. Figure 9(c) shows a calculated photon map corresponding to the parameters of Fig. 9(b). In the calculation, only the component of the electric field parallel to the polarization direction in the photon map (and, therefore, parallel to the plane of the image) is involved. The intensity distribution of the calculated image reproduces reasonably well the observed one.

Figure 10(a) shows line cuts of the photon maps along the polarization direction. It is readily seen that the intensity distribution of the photon map taken at $200 \mathrm{kV}$ extends farther outside the particle than that taken at $100 \mathrm{kV}$. The maximum of the intensity occurs near the particle edge for $200 \mathrm{kV}$, whereas it occurs inside from the particle edge for $100 \mathrm{kV}$. Figure 10(b) shows calculated intensity profiles of the dipole mode emission corresponding to those in Fig. 10(a). The theory [solid curves in Fig. 10(b)] explains the position of the maximum at $200 \mathrm{kV}$, which is very close to the particle 

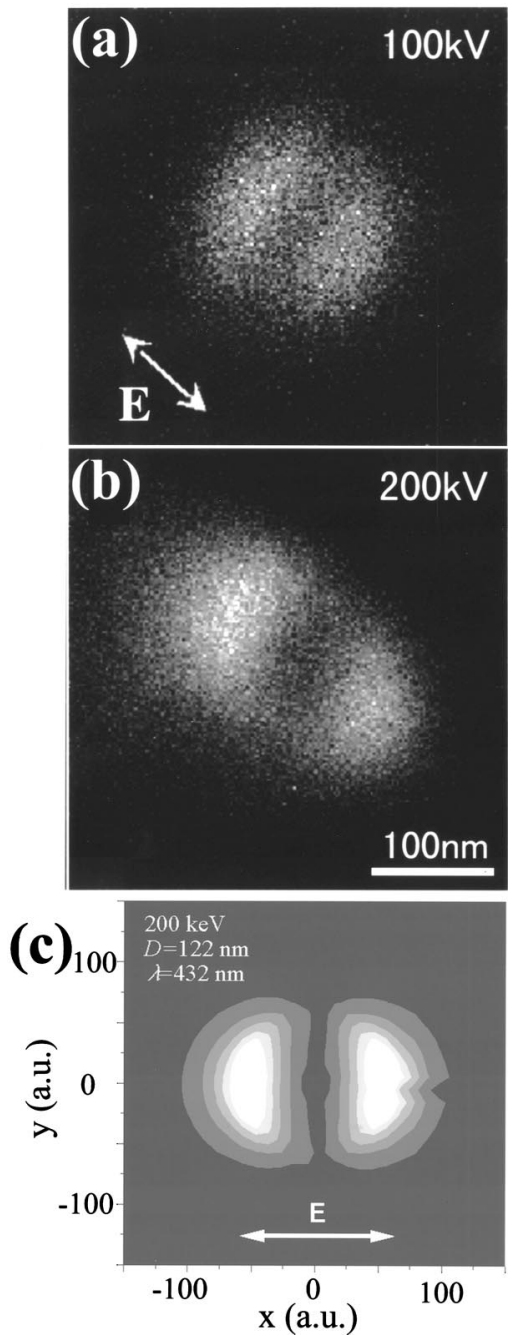

FIG. 9. Photon maps of a silver particle of diameter $D=122 \mathrm{~nm}$ taken for an electron accelerating voltage of (a) $100 \mathrm{kV}$ and (b) 200 $\mathrm{kV}$ with light corresponding to the peak wavelength of $432 \mathrm{~nm}$ (see Fig. 8). (c) Calculated photon map corresponding to (b). The light polarization is shown by arrows.

edge, although it fails to predict the position of the observed peak at $100 \mathrm{kV}$, farther inside the particle. Deviation from perfect spherical shape might be one reason for such a discrepancy.

The solid curves in Fig. 10(b) are obtained from the $x$ component of the far electric field (with $x$ perpendicular to the incident beam direction), whereas the dashed curves are calculated using both the $x$ and $z$ components, where the $z$ axis is taken parallel to the incident beam direction. The $z$ component of the electric field can also contribute to the emission intensity of $p$-polarized light after reflection by the ellipsoidal mirror. However, the solid curves agree better with the observed one. This can be due to the fact that the $z$ component of the field has a significant weight only at relatively large $\theta$ angles (see Fig. 1), for which the reflection efficiently of the ellipsoidal mirror that focuses the emitted light on the slit of the monochromater can be poor due to possible misalignment of the mirror or imperfection of the mirror plane.

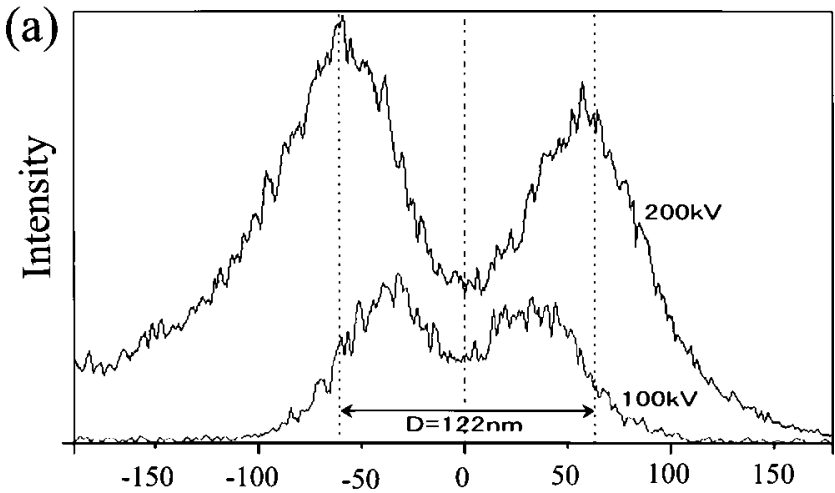

(b)

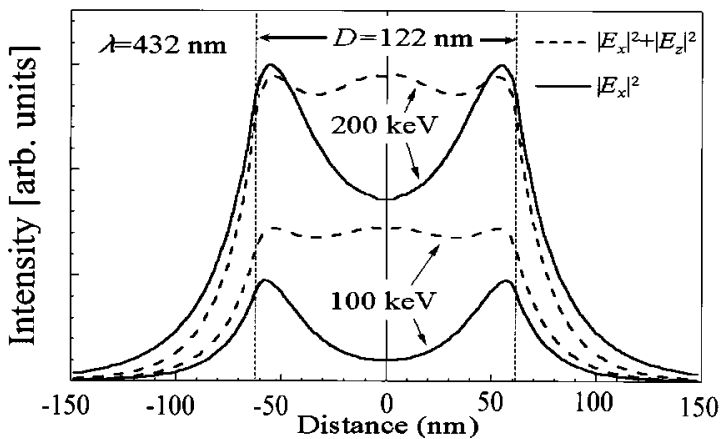

FIG. 10. (a) Line cuts of the photon maps of Figs. 9(a) and 9(b) along the polarization direction indicated by vector $\mathbf{E}$. (b) Intensity profiles calculated with the $x$ component of the radiation field (solid curves) and both the $x$ and $z$ components (dashed curves) for an electron accelerating voltage of 100 and $200 \mathrm{kV}$, where the $z$ direction is chosen along the electron beam direction (see text for further details).

Light emission is still generated even when the incident electron path is away from the particle edge by a finite distance. This is seen as a tail of the emission intensity outside the sphere edge. Since the modified Bessel function $K_{m}(\omega b / v \gamma)$ in Eq. (2) can be approximated by an exponentially decaying function at large distances, the interaction distance is characterized by the value $v \gamma / \omega$ (notice that the nonretarded characteristic distance is given by $v / \omega$ instead). The photon maps of Figs. 9(a) and 9(b) clearly show that the interaction distance becomes longer as the accelerating voltage increases from 100 to $200 \mathrm{kV}$. The ratio of impact parameters for which the argument of the modified Bessel function is the same at 100 and $200 \mathrm{kV}$ can be written

$$
\frac{b_{200}}{b_{100}}=\frac{(\beta \gamma)_{200}}{(\beta \gamma)_{100}}=1.49,
$$

where $\beta=v / c$, and this value is larger than the nonretarded value $\beta_{200} / \beta_{100}=1.27$ by the ratio of the Lorenz factors. The observed increase in the range of emission intensity away from the particle is qualitatively explained by this factor.

\section{CONCLUSIONS}

Photons emitted from submicron silver particles illuminated by fast electrons have been observed using a light detection system combined with a $200-\mathrm{kV}$ TEM. Focusing the 
electron beam on a given position on or near the particles, the recorded emission spectra (Figs. 2, 4, and 5) are seen to be characterized by several peaks that have been explained in terms of the excitation of collective plasmon modes.

In a scanning mode of operation, the collected photon emission has been used to create photon maps at fixed photon wavelengths (Figs. 3, 6, and 9), where each pixel in the image corresponds to a given position of the electron beam as it scans the particle region and the intensity of that pixel is made proportional to the photon flux for that particular beam position for the fixed photon wavelength. The photon maps observed at the wavelengths corresponding to the peak positions have permitted to conclude that the peaks are coming from the excitation of multipole modes with dipolar, quadrupolar, and haxapolar character.

The particles appear to have rather spherical shapes, so that the measured emission spectra and photon maps have been compared with calculations for photon emission from metallic particles induced by the passage of fast electrons. The agreement between theory and experiment is quite satisfactory (e.g., in the photon maps of Fig. 9). In particular, the photon energies corresponding to the multipole modes of order $l=1,2$, and 3 follow quite closely the theoretical dependence on particle diameter $D$ (Fig. 7), including retardation corrections that produce a significant decrease in the energy of a given mode with increasing $D$. The important role played by retardation is due to the fact that the particle diameters are comparable in magnitude to the wavelengths under consideration.

The dependence on the accelerating voltage has been examined as well, and it has been observed that the wavelengths of the peaks corresponding to the multipole modes are independent of the accelerating voltage, while the relative intensities of those peaks change with the electron velocity, as predicted by theory [Eq. (2)] (see Fig. 8).

Finally, it has been found that the intensity distribution in the photon maps extends further outside the particle edge as the accelerating voltage increases, in good agreement with the theory, although some discrepancy still exist between theory and experiment relative to the precise spatial position of the emission maxima. This discrepancy can originate in deviations from perfect particle sphericity and in the effect of the support of the particles. Further research in this direction is underway.

\section{ACKNOWLEDGMENTS}

The authors would like to thank Professor A. Howie for enjoyable and useful discussions on retardation effects. One of the authors (F.J.G.A.) acknowledges help and support from the University of the Basque Country and the Spanish Ministerio de Educación y Cultura (Fulbright grant No. FU-98-22726216). This work was supported by the New Frontier Program Grant-in-Aid for Scientific Research (No. 09NP1201).
${ }^{1}$ S. J. Smith and E. M. Purcell, Phys. Rev. 92, 1069 (1953); J. Urata, M. Goldstein, M. F. Kimmit, A. Naumov, C. Platt, and J. E. Walsh, Phys. Rev. Lett. 80, 516 (1998).

${ }^{2}$ F. J. García de Abajo, Phys. Rev. Lett. 82, 2776 (1999); Phys. Rev. E 61, 5743 (2000).

${ }^{3}$ N. Yamamoto and A. Toda, Scanning Microsc. 9, 669 (1995).

${ }^{4}$ N. Yamamoto, A. Toda, and K. Araya, J. Electron Microsc. 45, 64 (1996).

${ }^{5}$ N. Yamamoto, H. Sugiyama, and A. Toda, Proc. R. Soc. London, Ser. A 452, 2279 (1996).

${ }^{6}$ K. Araya and N. Yamamoto (unpublished) (1998).

${ }^{7}$ R. Fuchs and K. L. Kliewer, J. Opt. Soc. Am. 58, 319 (1968).

${ }^{8}$ P. E. Batson, Phys. Rev. Lett. 49, 936 (1982); Surf. Sci. 156, 720 (1985).

${ }^{9}$ D. Ugarte, C. Colliex, and P. Trebbia, Phys. Rev. B 45, 4332 (1992).
${ }^{10}$ Z. L. Wang and J. M. Cowley, Ultramicroscopy 21, 77 (1987); 21, 335 (1987); 21, 347 (1987); 23, 97 (1987).

${ }^{11}$ A. Howie and C. A. Walsh, Microsc. Microanal. Microstruct. 2, 171 (1991).

${ }^{12}$ T. L. Ferrell and P. M. Echenique, Phys. Rev. Lett. 55, 1526 (1985).

${ }^{13}$ P. M. Echenique, A. Howie, and D. J. Wheatley, Philos. Mag. B 56, 335 (1987).

${ }^{14}$ P. M. Echenique and J. B. Pendry, J. Phys. C 8, 2936 (1975).

${ }^{15}$ J. Aizpurua, A. Howie, and F. J. García de Abajo, Phys. Rev. B 60, 11149 (1999).

${ }^{16}$ F. Fujimoto and K. Komaki, J. Phys. Soc. Jpn. 25, 1679 (1968).

${ }^{17}$ F. J. García de Abajo and A. Howie, Phys. Rev. Lett. 80, 5180 (1998).

${ }^{18}$ F. J. García de Abajo, Phys. Rev. B 59, 3095 (1999).

${ }^{19}$ P. B. Johnson and R. W. Christy, Phys. Rev. B 6, 4370 (1972). 